\title{
Anisotropic Quintessence stars
}

\author{
Mehedi Kalam* \\ Department of Physics, Aliah University, Sector - V, Salt Lake, Kolkata - 700091, India \\ Farook Rahaman† \\ Department of Mathematics, Jadavpur University, Kolkata 700 032, West Bengal, India \\ Sajahan Molla团 \\ Department of Physics, Aliah University, Sector - V, Salt Lake, Kolkata, India \\ Sk. Monowar Hossein \\ Department of Mathematics, Aliah University, Sector - V, Salt Lake, Kolkata - 700091, India
}

(Dated: April 27, 2018)

\begin{abstract}
We propose a relativistic model for ,cs with the combination of an anisotropic pressure corresponding to normal matter and a quintessence dark energy having a characteristic parameter $\omega_{q}$ such that $-1<\omega_{q}<-\frac{1}{3}$. We discuss various physical features of the model and show that the model satisfies all the regularity conditions and can provide stable equilibrium configurations.

PACS numbers: 04.40.Nr, 04.20.Jb, 04.20.Dw
\end{abstract}

\section{INTRODUCTION}

Compact objects are of great interest for a long time. Theoretical analysis of superdense stars have been done by several authors [1 10]. Ruderman[11] show that nuclear matter may have anisotropic behaviors at a very high density $\left(\sim 10^{18} \mathrm{Kg} / \mathrm{m}^{3}\right)$. Consideration of an anisotropic behavior of the compact star leads to a realistic situation [12, 13]. Anisotropy in matter implies radial pressure $\left(p_{r}\right)$ is not equal to the tangential pressure $\left(p_{t}\right)$. As the density of a strange star exceeds the nuclear density, it is obvious that the pressure at the interior should be anisotropic [15, 18, 26]. It may occur for various reasons like existence of solid core, phase transition, presence of electromagnetic field etc. In recent past, L.Herrera and N.O. Santos [19] provided an exhaustive review on the subject of anisotropic fluids. More recently a comprehensive work on the influence of local anisotropy on the structure and evolution of compact object has been studied by L. Herrera et. al [20].

In recent WMAP measurement conclude that $73 \%$ of the universe is dark energy [16, 17]. Dark Energy theory is the most accepted one to explain the acceleration of the universe. It has some peculiar properties such as negative pressure and violation of the energy conditions. Current experimental data shows that pressure to density ratio $\omega$ is in the range $-1.38<\omega<-0.82$. In the present work, we consider models of compact stars containing not only ordinary matter but also a

\footnotetext{
*Electronic address: kalam@iucaa.ernet.in

${ }^{\dagger}$ Electronic address: rahaman@iucaa.ernet.in

‡Electronic address: sajahan.phy@gmail.com

$\S$ Electronic address: sami milu@yahoo.co.uk
}

quintessence matter having a characteristic parameter $\omega_{q}$ such that $-1<\omega_{q}<-\frac{1}{3}$. The presence of dark energy motivates us to consider the existing strange stars are a mixture of both ordinary matter and quintessence matter in different proportions. The study of such kind of mixed matter is now an interesting problem and some works has already been done on this direction [1, 1, , 8, 21].

Some important works was carried out with Krori and Barua [14] (KB)metric by Rahaman et al. [1] for singularity-free dark energy stars which represents an anisotropic compact stellar configuration. The same KB spacetime was used to study the strange stars by using the cosmological constant as a dark energy source by Kalam et al. [3] and Hossein et al. [4]. Moreover by using the same metric Kalam et al. [6] presents a interesting anisotropic strange star model.The beauty of the model is that the interior physical properties of the star solely depend on the central density of the matter distribution.

We would like to mention here the very recent work of Kalam et al. [5] where the authors have proposed a model for strange quark stars within the framework of MIT Bag model in Finch and Skea metric 22]. F.S. Lobo[7], R. Chan et al. 21], Egeland [9], Dymnikova 10] and many more have also studied stellar structure in different ways.

As mentioned above basically we have considered here a two fluids model for compact star with quintessence dark energy as one of the ingredients. Here we consider the same Finch and Skea metric and the solution satisfies all the energy conditions including TOV-equations. We also check the mass-radius relation, stability and surface red-shifts for our model and found that their behavior is well behaved.

The plan of the present paper is as follows: In Sec II 
we have provided the basic equations in connection to the proposed model for strange star of Finch and Skea metric.In Sec. III we dealt with the physical behavior of the star. Anisotropic behavior, Matching conditions, TOV equations, Energy conditions, Stability and MassEnergy relations are discussed in different Sub-sections. Concluding remarks are made in Sec. IV.

\section{QUINTESSENCE STAR MODEL}

We assume that the interior space-time of a quintessence star is described by the metric

$d s^{2}=-e^{\nu(r)} d t^{2}+\left(1+\frac{r^{2}}{R^{2}}\right) d r^{2}+r^{2}\left(d \theta^{2}+\sin ^{2} \theta d \phi^{2}\right)$,

where the metric function $\nu(r)$ is yet to be determined and $R$ is a constant. Consideration of such a space-time, describing a paraboloidal geometry, is not new in the analysis of relativistic compact stars 22].

Now we consider a compact star which contains a quintessence field and a normal matter producing an anisotropic pressure. Therefore, the Einstein equations can be written as

$$
G_{\mu \nu}=8 \pi\left(T_{\mu \nu}+\tau_{\mu \nu}\right)
$$

where $\tau_{\mu \nu}$ represents the energy momentum tensor of the quintessence-like field, which is characterized by a free parameter $\omega_{q}$ with the restriction $-1<\omega_{q}<-\frac{1}{3}$. According to Kiselev [23], the components of this tensor necessarily satisfy the conditions of additivity and linearity. Considering the different signatures used in the line elements, the components can be stated as follows:

$$
\begin{gathered}
\tau_{t}^{t}=\tau_{r}^{r}=-\rho_{q}, \\
\tau_{\theta}^{\theta}=\tau_{\phi}^{\phi}=\frac{1}{2}\left(3 \omega_{q}+1\right) \rho_{q} .
\end{gathered}
$$

However, the most general energy momentum tensor compatible with spherical symmetry is

$$
T_{\nu}^{\mu}=\left(\rho+p_{t}\right) u^{\mu} u_{\nu}-p_{t} g_{\nu}^{\mu}+\left(p_{r}-p_{t}\right) \eta^{\mu} \eta_{\nu}
$$

with $u^{\mu} u_{\mu}=-1$.

Einstein's field equations for the metric (1) accordingly are obtained as $(c=1, G=1)$

$$
\begin{aligned}
8 \pi\left(\rho+\rho_{q}\right) & =\frac{1}{R^{2}}\left(3+\frac{r^{2}}{R^{2}}\right)\left(1+\frac{r^{2}}{R^{2}}\right)^{-2},(6) \\
8 \pi\left(p_{r}-\rho_{q}\right)= & \left(1+\frac{r^{2}}{R^{2}}\right)^{-1}\left[\frac{\nu^{\prime}}{r}+\frac{1}{r^{2}}\right]-\frac{1}{r^{2}}(7) \\
8 \pi\left(p_{t}+\frac{\left(3 \omega_{q}+1\right)}{2} \rho_{q}\right)= & \left(1+\frac{r^{2}}{R^{2}}\right)^{-1}\left[\frac{\nu^{\prime \prime}}{2}+\frac{\nu^{\prime}}{2 r}+\frac{\nu^{\prime 2}}{4}\right] \\
& -\frac{1}{R^{2}}\left(1+\frac{r^{2}}{R^{2}}\right)^{-2}\left[1+\frac{\nu^{\prime} r}{2}\right](.8)
\end{aligned}
$$

To solve the above set of equations, we assume that the radial pressure of the stellar body is proportional to the matter density, i.e.

$$
p_{r}=m \rho
$$

where $\mathrm{m}(>0)$ is the equation of state parameter.

Now, from the metric(1) and equations (6)-(9), we get

$$
\begin{aligned}
\nu= & \frac{m}{2}\left[2 \ln \left(1+\frac{r^{2}}{R^{2}}\right)+\frac{r^{2}}{R^{2}}\right]+\ln \left(\frac{1}{R}\right)+\frac{r^{2}}{2 R^{2}} \\
& -8 \pi(1+m) \int r\left(1+\frac{r^{2}}{R^{2}}\right) \rho_{q} d r
\end{aligned}
$$

Now, we take the quintessence field as

$$
\rho_{q}=A(2 b-r) e^{r}
$$

where $\mathrm{A}$ is a constant of dimension Length ${ }^{-3}$ and b is the radius of the star.

Although the quintessence field has a repulsive nature, the real matter at the interior region of the stellar body dominates. When we go beyond from the centre of the star then quintessence field gradually increases. However, up to the boundary real matter dominates and stabilize the stellar structure (Fig. 1).

By taking the above consideration of the quintessence field we get

$$
\begin{aligned}
& \nu= \frac{m}{2}\left[2 \ln \left(1+\frac{r^{2}}{R^{2}}\right)+\frac{r^{2}}{R^{2}}\right]+\ln \left(\frac{1}{R}\right)+\frac{r^{2}}{2 R^{2}} \\
&+8 \pi(1+m) A e^{r}\left[\left(r^{2}-2 b r-2 r+2 b+2\right)\right. \\
&+\frac{1}{R^{2}}\left\{r^{4}-2(b+2) r^{3}\right. \\
&\left.\left.+6(b+2) r^{2}-12(b+2) r+12(b+2)\right\}\right] \\
& 8 \pi \rho= \frac{1}{R^{2}} \frac{\left(3+\frac{r^{2}}{R^{2}}\right)}{\left(1+\frac{r^{2}}{R^{2}}\right)^{2}}-8 \pi A(2 b-r) e^{r} \\
& 8 \pi p_{r}=\frac{1}{R^{2}} \frac{m\left(3+\frac{r^{2}}{R^{2}}\right)}{\left(1+\frac{r^{2}}{R^{2}}\right)^{2}}-8 \pi m A(2 b-r) e^{r} \\
& \quad-8 \pi A(2 b-r) e^{r} \frac{\left(3 \omega_{q}+1\right)}{2} \\
& 8 \pi p_{t}=\left(1+\frac{r^{2}}{R^{2}}\right)^{-1}\left[\frac{\nu^{\prime \prime}}{2}+\frac{\nu^{\prime}}{2 r}+\frac{\nu^{\prime 2}}{4}\right] \\
&\left(1+\frac{r^{2}}{R^{2}}\right) \\
&-2
\end{aligned}
$$




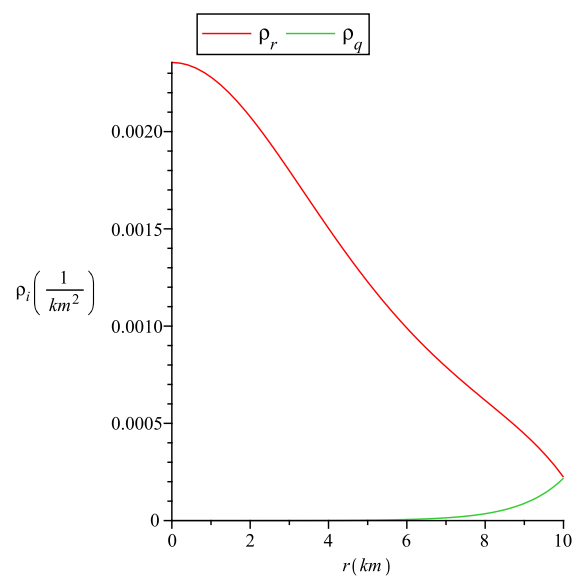

FIG. 1: Density comparison of real and quintessence matters at the stellar interior.

\section{PHYSICAL ANALYSIS}

We note that the physical behavior of the star depends on the constants $\mathrm{R}$ and $\rho_{q}$. Here, we need to put appropriate assumption to get the realistic model.In this section we will discuss the following features of our model :

\section{A. Anisotropic Behavior of the star}

From Eq. (13), the central and surface densities are obtained as

$$
\begin{aligned}
& \rho_{0}=\frac{3}{8 \pi R^{2}}-2 A b \\
& \rho_{b}=\frac{1}{8 \pi R^{2}}\left(3+\frac{b^{2}}{R^{2}}\right)\left(1+\frac{b^{2}}{R^{2}}\right)^{-2}-A b e^{b}
\end{aligned}
$$

where we have assumed that $b$ is the radius of the star and density due to quintessence field at the centre of the star, $\rho_{q}=2 \mathrm{Ab}$. Therefore, We can say that

$$
\rho_{0 e f f}=\frac{3}{8 \pi R^{2}}>0
$$

and

$$
\rho_{e f f}=\frac{1}{8 \pi R^{2}} \frac{\left(3+\frac{r^{2}}{R^{2}}\right)}{\left(1+\frac{r^{2}}{R^{2}}\right)^{2}}>0
$$

Now, we check whether at the centre the effective matter density dominates or not. Here, we see that

$$
\begin{aligned}
\frac{d \rho_{e f f}}{d r} & =-\frac{r\left(5+\frac{r^{2}}{R^{2}}\right)}{4 \pi R^{4}\left(1+\frac{r^{2}}{R^{2}}\right)^{3}}<0, \\
\frac{d \rho_{e f f}}{d r}(r=0) & =0 \\
\frac{d^{2} \rho_{e f f}}{d r^{2}}(r=0) & =-\frac{5}{4 \pi R^{4}}<0 .
\end{aligned}
$$

Clearly, at the centre, the effective density of the star is maximum and it decreases radially outward. Similarly, from Eq.(7), we get

$$
\frac{d p_{r e f f}}{d r}=-\frac{m r\left(5+\frac{r^{2}}{R^{2}}\right)}{4 \pi R^{4}\left(1+\frac{r^{2}}{R^{2}}\right)^{3}}<0
$$

Here, again at the centre $(r=0)$,

$$
\begin{aligned}
\frac{d p_{\text {reff }}}{d r}(r=0) & =0 \\
\frac{d^{2} p_{r e f f}}{d r^{2}}(r=0) & =-\frac{5 m}{4 \pi R^{4}}<0
\end{aligned}
$$

Therefore, at the centre, we also see that the effective radial pressure is maximum and it decreases from the centre towards the boundary. Thus, the effective energy density and the effective radial pressure are well behaved in the interior of the stellar structure. Variations of the effective energy-density and effective radial pressure have been shown in Fig. 2 and Fig. 3, respectively.

The anisotropic parameter $\Delta(r)=\frac{2}{r}\left(p_{t}-p_{r}\right)$ representing the anisotropic stress is shown in Fig. 4.

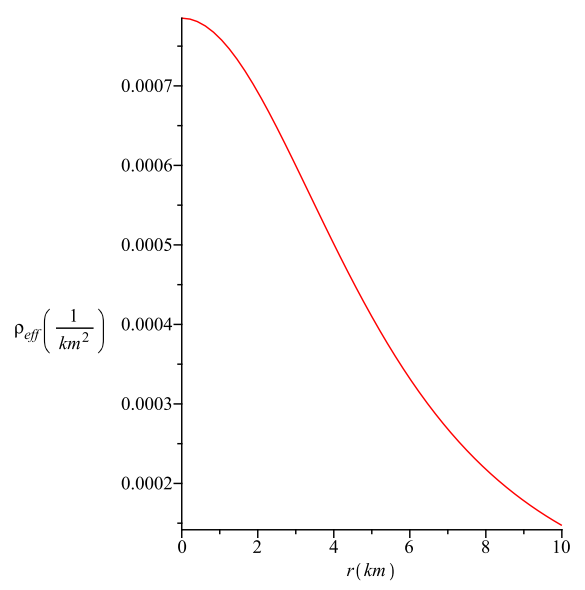

FIG. 2: Variation of the effective energy-density $(\rho)$ at the stellar interior.

\section{B. Matching Conditions}

Interior metric of the star should be matched to the Schwarzschild exterior metric at the boundary $(r=b)$.

$d s^{2}=-\left(1-\frac{2 M}{r}\right) d t^{2}+\left(1-\frac{2 M}{r}\right)^{-1} d r^{2}+r^{2}\left(d \theta^{2}+\sin ^{2} \theta d \phi^{2}\right)$

Assuming the continuity of the metric functions $g_{t t}, g_{r r}$ and $\frac{\partial g_{t t}}{\partial r}$ at the boundary, we get 


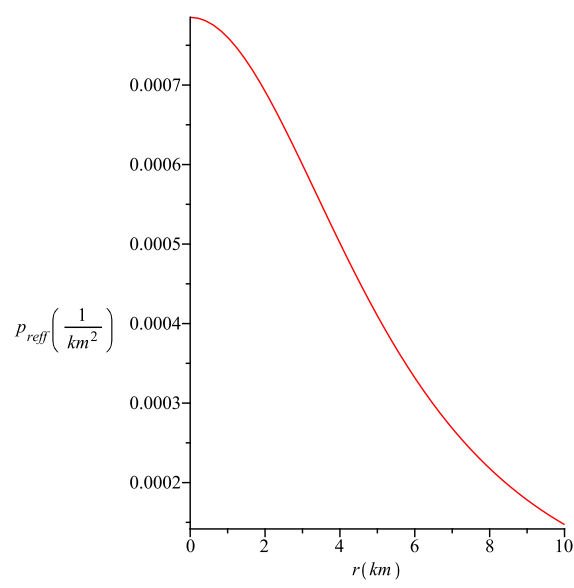

FIG. 3: Variation of the effective radial pressure $\left(p_{r}\right)$ at the stellar interior.

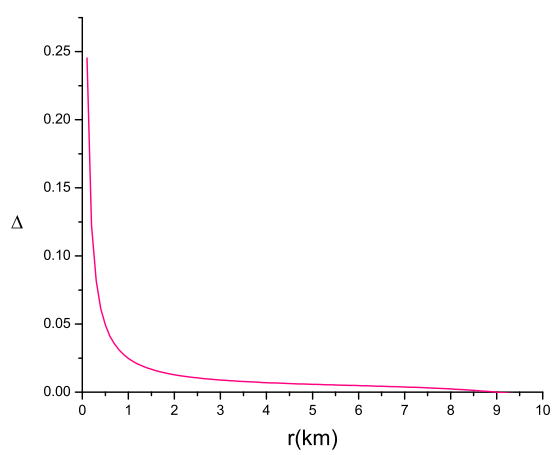

FIG. 4: Effective anisotropic behavior $\Delta(r)$ at the stellar interior.

$$
\begin{aligned}
\left(1+\frac{b^{2}}{R^{2}}\right)^{-1}= & 1-\frac{2 M}{b}, \\
\nu(r=b)= & \ln \left(1-\frac{2 M}{b}\right)=\frac{m}{2}\left[2 \ln \left(1+\frac{b^{2}}{R^{2}}\right)+\frac{b^{2}}{R^{2}}\right] \\
& +\ln \left(\frac{1}{R}\right)+\frac{b^{2}}{2 R^{2}}-8 \pi(1+m) A e^{b} \\
& {\left[\left(b^{2}-2\right)+\frac{1}{R^{2}}\left(b^{4}-2 b^{3}+12 b-24\right)\right](21) }
\end{aligned}
$$

We plot ( Fig. 5 ) the behaviors of pressure anisotropy, gravitational and hydrostatic forces at the stellar interior of $4 \mathrm{U} 1820-30$ which indicates the static equilibrium configurations do exist in the presence of pressure anisotropy, gravitational and hydrostatic forces.

From Eq. (20), we get the compactification factor as

$$
\frac{M_{e f f}}{b}=\frac{b^{2}}{2 R^{2}}\left(1+\frac{b^{2}}{R^{2}}\right)^{-1}
$$

Equation (21) yields the value of unknown constant A in terms of compactification factor, equation of state parameter and $b$, the radius of the star.

\section{Energy conditions}

For an anisotropic fluid distribution, the generalized TOV equation has the form

$$
\frac{d p_{r \text { eff }}}{d r}+\frac{1}{2} \nu^{\prime}\left(\rho_{\text {eff }}+p_{\text {reff }}\right)+\frac{2}{r}\left(p_{r \text { eff }}-p_{t \text { eff }}\right)=0 .
$$

Following León [25], we write the above equation as

$$
-\frac{M_{G}\left(\rho_{e f f}+p_{r e f f}\right)}{r^{2}} e^{\frac{\lambda-\nu}{2}}-\frac{d p_{r e f f}}{d r}+\frac{2}{r}\left(p_{t e f f}-p_{r \text { eff }}\right)=0,
$$

where $M_{G}(r)$ is the effective gravitational mass inside a sphere of radius $r$ and is given by

$$
M_{G}(r)=\frac{1}{2} r^{2} e^{\frac{\nu-\lambda}{2}} \nu^{\prime}
$$

where $e^{\lambda(r)}=1+\frac{r^{2}}{R^{2}}$

which can be derived from the Tolman-Whittaker formula and the Einstein's field equations. The modified TOV equation describes the equilibrium condition for the compact quintessence star subject to effective gravitational $\left(F_{g}\right)$ and effective hydrostatic $\left(F_{h}\right)$ plus another force due to the effective anisotropic $\left(F_{a}\right)$ nature of the stellar object as

$$
F_{g}+F_{h}+F_{a}=0,
$$

where,

$$
\begin{aligned}
F_{g}= & -\frac{1}{2}\left(\rho_{\text {eff }}+p_{r \text { eff }}\right)\left[\frac{m}{R^{2}} \frac{3 r+\frac{r^{3}}{R^{2}}}{1+\frac{r^{2}}{R^{2}}}+\frac{r}{R^{2}}\right. \\
& -8 \pi A(1+m) e^{r}\left(2 b r-r^{2}+\frac{2 b r^{3}}{R^{2}}-\frac{r^{4}}{R^{2}}\right)(27) \\
F_{h}= & -\frac{d p_{r \text { eff }}}{d r} \\
F_{a}= & \frac{2}{r}\left(p_{t \text { eff }}-p_{r \text { eff }}\right)
\end{aligned}
$$

All the energy conditions, namely, null energy condition(NEC), weak energy condition(WEC), strong energy condition(SEC) and dominant energy condition(DEC), are satisfied. In our model, we get the following energy conditions at the centre $(r=0)$ :

(i) NEC: $p_{0 \text { eff }}+\rho_{0 \text { eff }} \geq 0$,

(ii) WEC: $p_{0}$ eff $+\rho_{0}$ eff $\geq 0, \quad \rho_{0}$ eff $\geq 0$,

(iii) SEC: $p_{0 \text { eff }}+\rho_{0}$ eff $\geq 0, \quad 3 p_{0}$ eff $+\rho_{0}$ eff $\geq 0$,

(iv) DEC: $\rho_{0}$ eff $>\mid p_{0}$ eff $\mid$. 


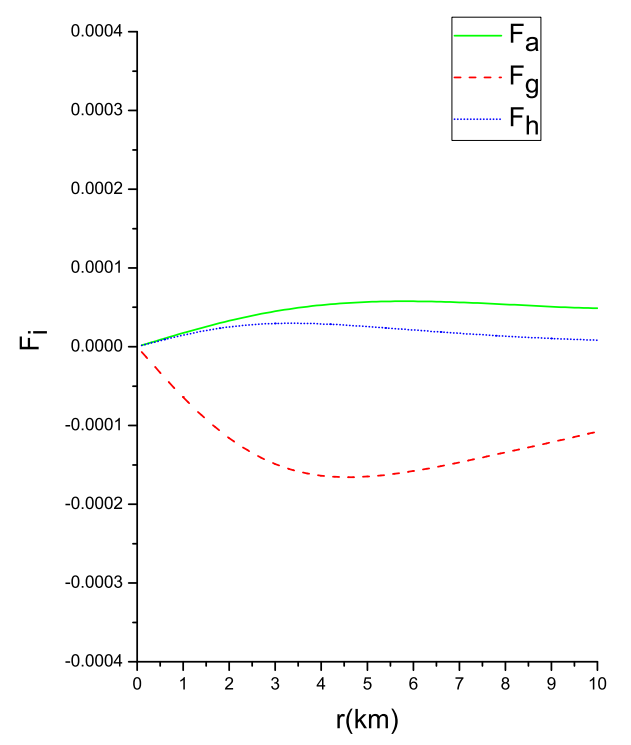

FIG. 5: Behaviors of pressure anisotropy, gravitational and hydrostatic forces at the stellar interior of $4 \mathrm{U}$ 1820-30.

\section{E. Stability}

For a physically acceptable model, one expects that the velocity of sound should be within the range $0 \leq v_{s}^{2}=$ $\left(\frac{d p}{d \rho}\right) \leq 1[26,27]$. In our case, we have

$$
v_{s r}^{2}=\frac{1}{3}
$$

We plot the radial and transverse sound speeds in Fig.6 which shows that these parameters satisfy the inequalities $0 \leq v_{s r}^{2} \leq 1$ and $0 \leq v_{s t}^{2} \leq 1$ everywhere within the stellar object. We also note that $v_{s t}^{2}-v_{s r}^{2} \leq 1$. Since, $0 \leq v_{s r}^{2} \leq 1$ and $0 \leq v_{s t}^{2} \leq 1$, therefore, $\left|v_{s t}^{2}-v_{s r}^{2}\right| \leq 1$. In Fig.7, we have plotted $\left|v_{s t}^{2}-v_{s r}^{2}\right|$. According to Herrera's Herrera [26] cracking (or overturning) theorem, these results show that the star model is stable. [ Herrera's Herrera [26] cracking (or overturning) theorem states that the region for which radial speed of sound is greater than the transverse speed of sound is a potentially stable region. ]

\section{F. Mass-Radius relation}

In this section, we study the maximum allowable massradius ratio in our model. According to Buchdahl [24], for a static spherically symmetric perfect fluid allowable mass-radius ratio is given by $\frac{2 M}{R}<\frac{8}{9}$ for a more generalized expression for the same see mass in terms of the

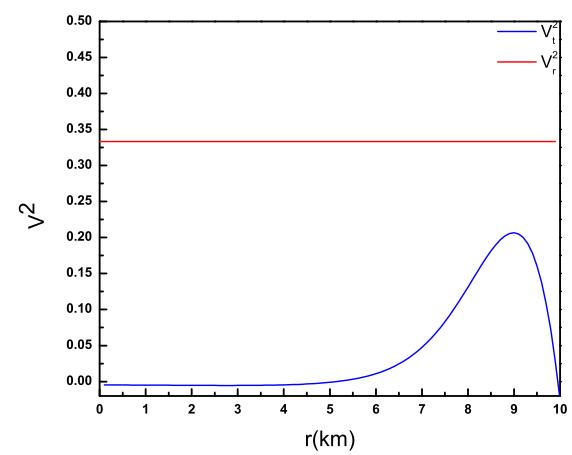

FIG. 6: Variation of the radial and transverse sound speed of $4 \mathrm{U} 1820-30$.

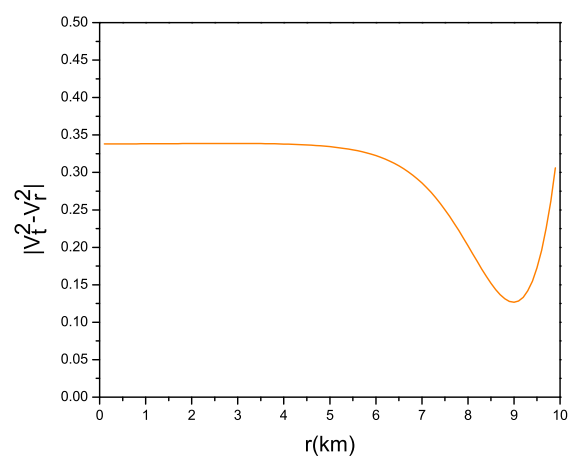

FIG. 7: Variation of $v_{s t}^{2}-v_{s r}^{2}$ of strange star $4 \mathrm{U}$ 1820-30.

effective energy density $\rho_{\text {eff }}$ can be expressed as

$$
M_{e f f}=4 \pi \int_{0}^{b} \rho_{e f f} r^{2} d r=\frac{b}{2}\left[\frac{\frac{b^{2}}{R^{2}}}{1+\frac{b^{2}}{R^{2}}}\right]
$$

We note that a constraint on the maximum allowed mass-radius ratio in our case is similar to the isotropic fluid sphere, i.e., $\frac{\text { mass }}{\text { radius }}<\frac{4}{9}$ as obtained earlier. The compactness of the star is given by

$$
u=\frac{M_{e f f}(b)}{b}=\frac{1}{2}\left[\frac{\frac{b^{2}}{R^{2}}}{1+\frac{b^{2}}{R^{2}}}\right]
$$

The surface red-shift $\left(Z_{s}\right)$ corresponding to the above compactness $(u)$ is obtained as

$$
1+Z_{s}=[1-(2 u)]^{-\frac{1}{2}}
$$

where

$$
Z_{s}=\sqrt{1+\frac{b^{2}}{R^{2}}}-1
$$

Thus, the maximum surface red-shift for the quintessence anisotropic stars of different radius could be found very easily ( Fig. 8 ). 


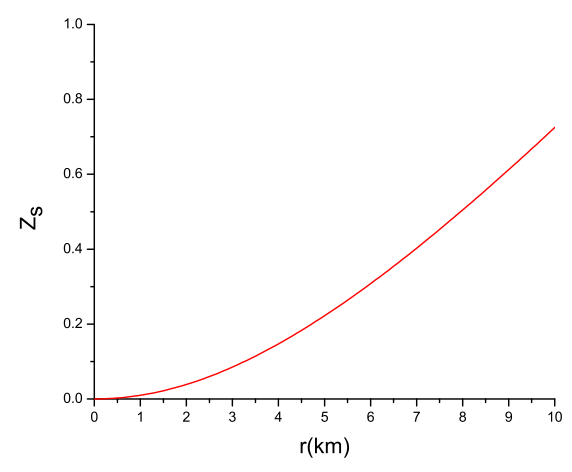

FIG. 8: Variation of the red-shift function of strange star $4 \mathrm{U}$ $1820-30$

\section{CONCLUSION}

In the present work, we investigate the nature of the quintessence type star by taking the Finch-Skea metric which describes the paraboidal geometry. In general compact stars, due to their high density, becomes anisotropic in nature. That's why we consider the anisotropic behavior of the star to make more generalized model.As the structure of these star are till not known, we assume that the interior stellar structure may constituted not only ordinary matter but also with a quintessence dark energy having a characteristic parameter $\omega_{q}$ such that $-1<\omega_{q}<-\frac{1}{3}$. The quintessence dark energy seems to be responsible for the accelerated expansion of the universe, therefore, inclusion of the quintessence dark energy in the interior of the highly compact stars is justified. The presence of dark energy motivates us to consider the existing strange stars are a mixture of both ordinary matter and quintessence dark energy in different proportions. We all know that quintessence has a repulsive nature. But, the real matter at the central region of the stellar body dominates to make the effective energy density to be positive. But when we go beyond from the centre of the star then quintessence field gradually increases. However, up to the boundary real matter dominates and stabilize the stellar structure. In conclusion, we can say that incorporation of quintessence matter with the real one describes the well-known compact stars (e.g. neutron stars,white dwarf stars, $4 \mathrm{U}$ 1820-30, Her X-1, SAX J 1808.4-3658 etc.) in a good manner in all respects.

\section{Acknowledgments}

MK, FR gratefully acknowledge support from IUCAA, Pune, India under Visiting Associateship under which a part of this work was carried out. SMH is thankful to IUCAA also for giving him an opportunity to visit IUCAA where a part of this work was carried out. FR is also thankful to UGC for providing financial support under Research Award Scheme. We are thankful to the referee for informing some of the important references.
[1] F. Rahaman et al., Gen. Rel ativ. Gravit. 44, 107 (2012).

[2] F. Rahaman et al., Eur. Phys. J. C 72, 2071 (2012).

[3] M. Kalam et al., Eur. Phys. J. C 72, 2248 (2012).

[4] Sk. M. Hossein et al, Int. J. Mod. Phys. D 21, 1250088 (2012).

[5] M. Kalam et al., arXiv: 1205.6795[gr-qc](to appear in Int.J.Theor.Phys.).

[6] M. Kalam et al., Eur. Phys. J. C 73, 2409 (2013).

[7] F. Lobo, Class. Quantum. Grav. 23, 1525 (2006).

[8] K. Bronnikov and J.C. Fabris, Phys. Rev. Lett. 96, 251101 (2006).

[9] E. Egeland, Compact Star, Trondheim, Norway (2007).

[10] I. Dymnikova, Class. Quantum. Gravit. 19, 725 (2002).

[11] R. Ruderman, Rev. Astr. Astrophys. 10, 427 (1972).

[12] V. Varela et al., Phys. Rev. D 82, 044052 (2010).

[13] F. Rahaman et al., Phys. Rev. D 82, 104055 (2010).

[14] K.D. Krori and J. Barua, J. Phys. A.: Math. Gen. 8, 508 (1975).

[15] R.L. Bowers and E.P.T. Liang, Astrophys. J. 188657
(1974).

[16] S. Perlmutter et al, Nature 391, 51 (1998).

[17] A.G.Riess et al, Astrophys. J. 607, 665 (2004).

[18] A.I. Sokolov, JETP 52, 575 (1980).

[19] L.Herrera and N.O. Santos, Phys.Report. 286, 53 (1997)

[20] L. Herrera, A. Di Prisco, J. Martin, J. Ospino, N.O.Santos, O. Troconis,, Phys. Rev. D 69, 084026, (2004) (1997)

[21] R. Chan et al, Gen. Relativ. Gravit. 41, 1835 (2009).

[22] Finch M. R. and Skea J. E. F., Class. Quantum. Grav., 6, 467 (1989)

[23] V.V. Kiselev, Class. Quantum. Grav., 20, 1187 (2003)

[24] Buchdahl, H. A., Phys. Rev., 116, 1027 (1959)

[25] León J. P. de, Gen. Relativ. Grav., 25, 1123 (1993)

[26] Herrera L., Phys. Lett. A, 165, 206 (1992)

[27] Abreu H., Hernandez H. and Nunez L. A., Class. Quantum. Grav., 24, 4631 (2007) 